This item is the archived peer-reviewed author-version of:

\title{
Air- and water-resistant noble metal coated ferromagnetic cobalt nanorods
}

\section{Reference:}

Lentijo-Mozo Sergio, Tan Reasmey P., Garcia-Marcelot Cécile, Altantzis Thomas, Bals Sara, Van Tendeloo Gustaaf, et al..- Airand water-resistant noble metal coated ferromagnetic cobalt nanorods

ACS nano - ISSN 1936-0851 - 9:3(2015), p. 2792-2804

Full text (Publishers DOI): http://dx.doi.org/doi:10.1021/nn506709k

To cite this reference: http://hdl.handle.net/10067/1253800151162165141 


\section{Air- and Water-Resistant Noble-Metal Coated \\ Ferromagnetic Cobalt Nanorods}

Sergio Lentijo-Mozo ${ }^{1}$, Reasmey P. Tan ${ }^{1}$, Cécile Garcia-Marcelot ${ }^{1,2}$, Thomas Altantzis ${ }^{3}$, PierFrancesco Fazzini $^{1}$, Teresa Hungria ${ }^{1}$, Benoit Cormary ${ }^{1}$, James R. Gallagher ${ }^{4}$, Jeffrey T. Miller ${ }^{4}$,

Herve Martinez ${ }^{5}$, Stefan Schrittwieser ${ }^{6}$, Joerg Schotter ${ }^{6}$, Marc Respaud ${ }^{1}$, Sara Bals ${ }^{3}$, Gustaaf Van Tendeloo $^{3}$, Christophe Gatel $^{2}$, and Katerina Soulantica ${ }^{1 *}$

1 Laboratoire de Physique et Chimie des Nano-objets (LPCNO), Université de Toulouse; INSA, UPS, CNRS, 135 avenue de Rangueil, 31077 Toulouse (France)

2 Centre d'Elaboration de Matériaux et d'Etudes Structurales (CEMES-CNRS), 29 rue Jeanne Marvig, B.P. 94347, 31055 Toulouse (France)

3 Electron Microscopy for Materials Research (EMAT), University of Antwerp, Groenenborgerlaan 171, 2020 Antwerp, (Belgium)

4 Chemical Science and Engineering Division, Argonne National Laboratory, 9700 S Cass Ave., Argonne, IL, 60439 (USA)

5 IPREM-ECP CNRS UMR 5254, Université de Pau, Hélioparc Pau Pyrénées, 2 av. Pierre Angot, 64053 Pau Cedex 9, (France) 
6 Molecular Diagnostics, AIT Austrian Institute of Technology, Vienna, Donau City Strasse 1, 1220 Vienna, (Austria)

KEYWORDS : Core-shell, ferromagnetic, nanorods, cobalt, water resistant.

\begin{abstract}
Cobalt nanorods possess ideal magnetic properties for applications requiring magnetically hard nanoparticles. However, their exploitation is undermined by their sensitivity towards oxygen and water, which deteriorates their magnetic properties. The development of a continuous metal shell inert to oxidation could render them stable, opening perspectives not only for already identified applications but also for uses in which contact with air and/or aqueous media is inevitable. However, the direct growth of a conformal noble metal shell on magnetic metals is a challenge. Here, we show that prior treatment of Co nanorods with a tin coordination compound is the crucial step, that enables the subsequent growth of a continuous noble metal shell on their surface, rendering them air- and water-resistant, while conserving the monocrystallity, metallicity and the magnetic properties of the Co core. Thus, the as-synthesized core-shell ferromagnetic nanorods combine high magnetization and strong uniaxial magnetic anisotropy, even after exposure to air and water, and hold promise for successful implementation in in vitro bio-diagnostics requiring probes of high magnetization and anisotropic shape.
\end{abstract}


Magnetic nanocrystals, either simple or multicomponent prepared by solution chemical methods can be exploited in very diverse technological domains. As a result, an important research activity in nanoscience concerns the development of magnetic nano-objects. ${ }^{1-5}$ Among them, metallic magnetic nanocrystals of the $3 \mathrm{~d}$ series such as Fe and Co display much higher magnetization than their oxide counterparts. This potential advantage, exploitable in many technologically important domains, is however undermined by their increased reactivity towards air and water which readily converts them to oxides and/or hydroxides. In particular, cobalt nanoparticles are characterized by a high magnetic moment that guarantees an efficient response to an externally applied magnetic field and a magnetic anisotropy that can be tuned through modification of their size, shape and crystal structure. ${ }^{6}$ Anisotropically shaped Co nanocrystals such as nanorods or nanowires are ideal candidates for applications concerning information storage and permanent magnets. ${ }^{7,8}$ Metallic Co single-crystalline nanorods (Co-NRs) of hexagonal close packed $(h c p)$ structure, prepared by the reduction of an organometallic or coordination Co precursor under hydrogen, ${ }^{9,10}$ exhibit particularly attractive magnetic properties $^{11}$ for implementation in domains requiring materials with both high magnetization and high coercivity. ${ }^{7,8,12}$ An efficient protection against oxidation of these nanorods is crucial for an implementation in all identified nanotechnology domains, and furthermore, it would open the way to applications not envisaged up to now. Protection of air-sensitive metallic magnetic nanoparticles against oxidation can be achieved through an efficient coating by an adequate shell. Metal oxides, polymers, silica, carbon and noble metals have been employed as shell materials and in a few cases a protection against oxidation has been reported, ${ }^{3,13-16}$ however, airand water-resistant ferromagnetic nanorods with a high magnetic moment have not been reported until now. 
A noble metal shell may not only act as an efficient barrier to oxidation, but it may also add its specific properties to those of the core, or become a platform for further surface functionalization. ${ }^{3,17-19}$ Gold is an ideal shell material because it is chemically stable, optically active and can be easily bio-functionalized. Platinum is also chemically inert, and despite the fact that its optical properties are less interesting compared to those of $\mathrm{Au}$, it is a highly active metal in catalysis. $^{20-23}$

Seeded-growth is the most efficient strategy for the synthesis of core-shell nanocrystals. ${ }^{24-26}$ The development of a conformal shell of a material on a seed nanocrystal depends on many parameters, such as the lattice mismatch between the core and the shell, the electronegativity, and the relative size of the atoms, which affect the interplay between surface and interface energies, and therefore, the final result. ${ }^{24-29}$ For colloidal nanocrystals, the presence of ligands, necessary to ensure a good dispersion in solution, may substantially alter surface and interfacial energies, but may also hamper seed-surface accessibility, preventing a homogeneous shell formation. ${ }^{29,30}$ It is furthermore important to stress that when noble metal ions come into contact with nanocrystals of a less noble metal, galvanic displacement may occur, partially dissolving the seed and reducing its dimensions. ${ }^{24,31}$ Some literature reports describe Co@Au and Co@Pt core@shell superparamagnetic spherical nanoparticles, ${ }^{31-33}$ but Co@M nanorods ( $\mathrm{M}=$ noble metal) have not been reported so far.

The growth of a conformal shell on nanorods is more complicated in comparison to isotropic nanocrystals, since it requires a homogeneous "wetting” of the various crystal facets despite their different reactivities. ${ }^{24,29}$ Furthermore the different crystal structures of the core and the shell, $h c p$ for Co nanorods and face centered cubic $(f c c)$ for the noble metal is an additional parameter 
that does not promote the formation of extended interfaces and therefore conformal shell formation.

Cobalt is not a target metal for in vivo biomedical applications due to its toxicity. However, the field of in vitro bio-diagnostics does not suffer from this factor and cobalt could respond to the specific needs of targeted biomedical applications, such as the point-of-care (POC) magnetooptical in vitro detection of important biomarkers in physiological liquid samples. ${ }^{34,35}$ Therefore, the growth of a protective shell would open the way to the successful implementation of highly performant, albeit up to now inexploitable in biomedical diagnosis, magnetic metallic nanoparticles. Apart from this specific application, protection of the Co core will also facilitate the implementation of such core-shell nanorods in magnetic recording, ${ }^{36}$ magnetically actuated liquid crystals, ${ }^{37}$ or ferrofluids. ${ }^{38}$ 


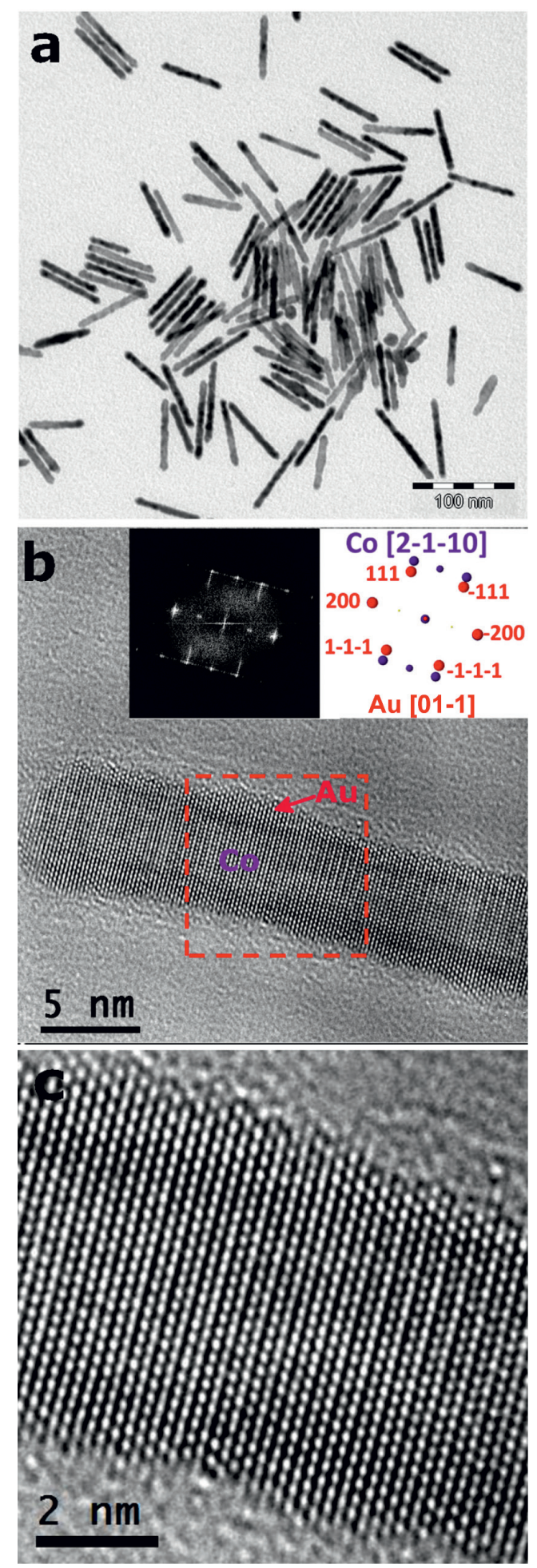

Figure 1. (a) Low magnification TEM image (scale bar: $100 \mathrm{~nm}$ ). (b) HRTEM image of a Co@SnAu nanorod, showing the core@shell structure. In the inset we present the fast Fourier transform (FFT) on the whole image showing the epitaxial relationship between the two lattices 
(scale bar: $5 \mathrm{~nm}$ ). (c) Magnification of the area marked by the red rectangle in b (scale bar: 2 $\mathrm{nm})$.

Here we show that whereas direct noble metal deposition on Co-NRs fails to form a continuous shell, a simple treatment of the Co-NRs with a Sn(II) coordination compound enables the subsequent development of a continuous noble metal shell of Au and Pt on Co-NRs, rendering them air- and water-resistant. ${ }^{39}$ The present method is expected to be generally applicable to metallic magnetic nanoparticles of various shapes, not only for protection purposes but also for obtaining performant and cost-efficient core-shell catalysts. ${ }^{40,41}$

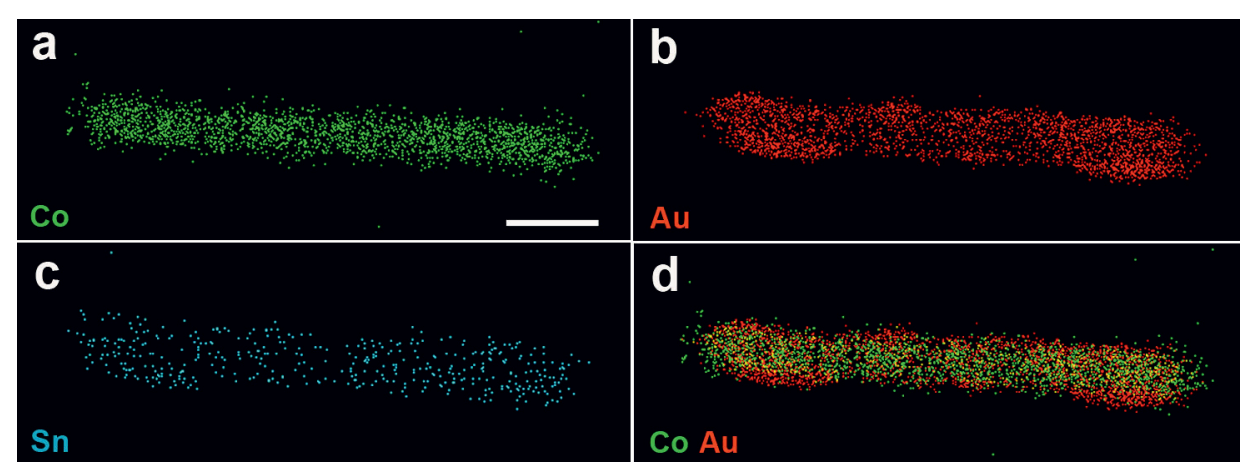

Figure 2. STEM-EDX maps showing the elemental distribution separately (a-c), and for the pair Co-Au (d) (Co: green, Au: red, Sn: blue). Scale bar for all EDX maps is $10 \mathrm{~nm}$.

\section{RESULTS AND DISCUSSION}

Gold-tipped as well as gold decorated Co-NRs have already been synthesized by using CoNRs as seeds. The preferential binding of surfactants on the lateral surfaces of the nanorods, drives Au growth selectively to the tips, where surfactant coverage is less efficient. Removing ligand excess from the entire surface, allows the non-selective decoration of the whole nanorod surface. ${ }^{42}$ Either when it is present on the apexes, or on the lateral nanorod sides, Au is epitaxially grown on the Co-NRs. Based on these results, we tried to develop a gold shell 
surrounding preformed Co-NRs, prepared in the presence of hexadecylamine (HDA) and lauric acid, by slight modifications of an already published method ${ }^{10}$ (see details in Supporting Information). However, all attempts have failed to cover the Co surface (Supporting Figure S1). In the best cases, where Au deposition was not limited to the tip area, a growth mode analogous to a Volmer-Weber epitaxial growth mode takes place, ${ }^{43,44}$ favoring small Co-Au interfaces instead of extended interfaces required for the formation of a complete shell.

Platinum is also resistant towards oxidation and has the advantage of being miscible with Co. Thus, provided that mild temperatures are employed during Pt overgrowth in order to avoid alloy formation, the growth of a continuous Pt shell around Co should be more favorable than in the case of $\mathrm{Au}$. We attempted to grow Pt directly on Co-NRs by reducing the organometallic precursor $\left[\mathrm{PtMe}_{2} \mathrm{COD}\right](\mathrm{Me}=$ methyl; $\mathrm{COD}=$ cyclooctadiene $)$ in the presence of HDA as a nanorod dispersant. Under all reaction conditions employed, a continuous Pt layer was not achieved. Independent Pt nucleation in solution was dominant, and in some cases we detected Pt "patches" on Co (Supporting Figure S2). It is thus possible, that surface accessibility, due to the presence of native ligands and the excess of HDA, hampers the access of the incoming metal to the Co-NRs surface. However, the presence of HDA is necessary in order to guarantee a good dispersion of the nanorod seeds in solution.

In order to overcome these obstacles, we have undertaken a surface modification of the CoNRs by introducing a buffer layer that could remediate interfacial strain and/or surface chemistry issues responsible for the full-shell formation failure. $\left[\left\{\mathrm{Sn}\left(\mathrm{NMe}_{2}\right)_{2}\right\}_{2}\right]$ can be reduced to metallic tin, which can form alloys with all three metals (Co, Au, Pt). ${ }^{45-48}$ A buffer layer with a gradient composition, in which the three elements are easily accommodated at the interface, could facilitate the development of a conformal metal shell..$^{49,50}$ Additionally, $\left[\left\{\mathrm{Sn}\left(\mathrm{NMe}_{2}\right)_{2}\right\}_{2}\right]$ acting 
as a ligand through the amide nitrogen, could displace the native laurate or HDA ligands present on the seed nanorod, ${ }^{10}$ which seem to hinder the interaction of the cobalt surface with the incoming noble metal. Finally, Sn(II) can be reduced to $\mathrm{Sn}(0)$ or oxidized to Sn(IV) in redox reactions as required, during the different stages of the shell growth.

Thus, Co-NRs were mixed with a $\left[\left\{\mathrm{Sn}\left(\mathrm{NMe}_{2}\right)_{2}\right\}_{2}\right]$ solution and HDA, and stirred at room temperature, in order for the tin compound to interact with the nanorod surface. [ $\left.\mathrm{AuClPPh}_{3}\right]$ $\left(\mathrm{PPh}_{3}=\right.$ triphenylphosphine) was then added, and the solution was heated under $\mathrm{H}_{2}$. The $\mathrm{Au}$ growth step was repeated once (See Supporting Information). Transmission Electron Microscopy (TEM) observations (Figure 1a) show that the resulting nano-objects are different than the CoAu structures prepared under exactly the same conditions, but without [ $\left.\left\{\mathrm{Sn}\left(\mathrm{NMe}_{2}\right)_{2}\right\}_{2}\right]$ (Supporting Figure S1d). 

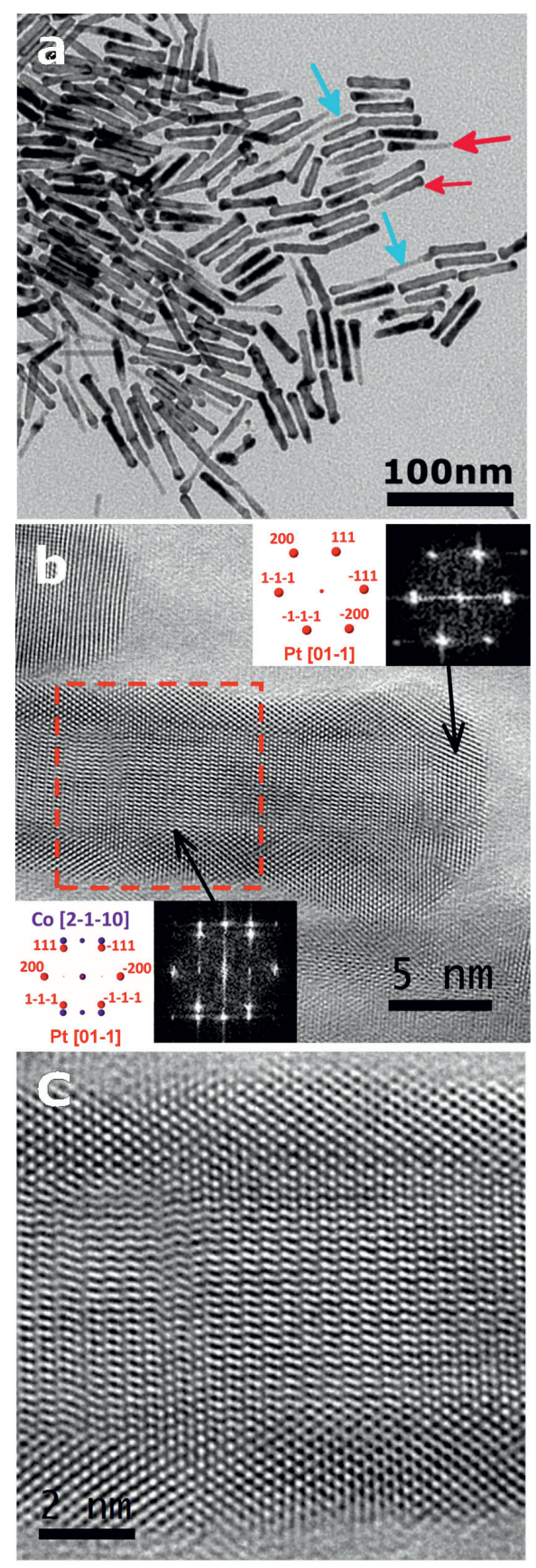

Figure 3. (a) Low magnification TEM image of Co@SnPt nanorod sample. The red arrows indicate rods where Co is not completely covered by the shell. Blue arrows indicate nanorods for which no shell can be discerned. (b) HRTEM image of an isolated nanorod. In the insets we present FFTs of the areas indicated by the arrows. The growth orientation is different on the 
lateral sides and on the tips. (c) Magnification of the area marked by the red rectangle in b (scale bar: $2 \mathrm{~nm})$.

Measurements of the nanorod dimensions before and after the reaction indicate that growth of a thin shell of less than $1 \mathrm{~nm}$ thickness has taken place (dimensions are given in Methods). High resolution TEM (HRTEM) observations reveal the presence of a thin layer surrounding the CoNR (Figure 1b,c). As expected, the Co core retains its $h c p$ crystal structure. Despite the fact that the shell layer thickness is not uniform, as shown further by the High-Angle Annular Dark-Field Scanning Transmission Electron Microscopy (HAADF-STEM) and complementary HRTEM images of Co@SnAu nanorods (Suppopting Figure S3), the shell can be considered as monocrystalline since the HRTEM images always reveal the same orientation relationships. The symbol used for the shell, corresponds to the order of material deposition on the Co core. The shell lattice parameters indeed correspond to Au, which has grown epitaxially on Co. The epitaxial relationship between Co and Au is as follows: Co[2-1-10](01-10) // Au[110](110) indicating that the interface plane is parallel to the (2-110) Co plane and (110) Au plane.$^{51}$ The lattice misfit $\mathrm{dAu}_{\mathrm{xyz}}-\mathrm{dCo}{ }_{\mathrm{abcd}} / \mathrm{dCo}{ }_{\mathrm{abcd}}$, referred to the Co bulk lattice, and based on lattice site coincidence relationships is $0.5 \%$, considering the inter-planar distances $\mathrm{d}_{2-1-10}$ for Co and $\mathrm{d}_{220}$ for $\mathrm{Au}$, and $-2.7 \%$ with $\mathrm{d}_{0001}$ for Co and $\mathrm{d}_{200}$ for Au.

The opportunity to add plasmonic properties to the hybrid nano-object led us to try to increase the layer thickness by repetitive Au depositions, which resulted in preferential Au growth on thicker shell areas, without however increasing significantly the thickness of the thinner areas (Supporting Figure S4a). The UV-Vis absorbance spectrum obtained from this sample shows a large resonance at about $570 \mathrm{~nm}$ which presumably corresponds to the plasmon resonance from 
thicker roughly spherical Au domains (Supporting Figure S4b). This growth mode is analogous to the Stranski-Krastanov heteroepitaxial growth mode observed in physical vapor deposition systems. ${ }^{29,43,44}$ Whereas the positive effect of Sn on the continuous Au shell growth is clear, the presence of Sn on the nanorods could not be revealed by HRTEM experiments. In order to detect the Sn and have an insight into the element distribution within the core-shell nanorods, STEMEnergy Dispersive X-ray (STEM-EDX) experiments were performed on isolated nano-objects by using a ChemiSTEM system (Figure 2a-d). From these images we can clearly see that both Au and $\mathrm{Sn}$ are present and homogeneously distributed in the same spatial region and there is no indication that the presence of Sn is limited to a layer between Co and Au.

A similar treatment of the Co-NRs with $\left[\left\{\mathrm{Sn}\left(\mathrm{NMe}_{2}\right)_{2}\right\}_{2}\right]$ followed by three successive Pt deposition steps (See Supporting Information), leads to the nanorods presented in Figure 3. The TEM images show that most of the nanorods are covered by a thin layer (Figure 3a). However, not all of them are uniformly coated. We can distinguish specimens of variable shell thickness and nanorods that appear to be completely bare, as indicated by red and blue arrows, respectively. Supplementary Pt depositions take place preferentially on already covered Pt-areas further increasing the existing thickness difference between covered and uncovered areas (Supporting Figure S5). Obviously, once the first layers of Pt deposited, the incoming Pt preferentially deposits on the already formed Pt shell. HRTEM studies (Figure 3b,c) show that Pt also grows epitaxially on the nanorod surface. The epitaxial relationship between Co and Pt on the lateral sides is: $\mathrm{Co}[2-1-10](01-10) / / \mathrm{Pt}[110](110)$, and on the tips $\mathrm{Co}[2-1-10](0001) / /$ $\mathrm{Pt}[110](111){ }^{51}$ The lattice misfit considering only the lateral planes of the nanorods, referred to 
the Co bulk lattice, and based on lattice site coincidence relationships is $-3.45 \%$ considering the inter-planar distances $\mathrm{d}_{2-1-10}$ for Co and $\mathrm{d}_{220}$ for Pt, and -6.75\% with $\mathrm{d}_{0001}$ for Co and $\mathrm{d}_{200}$ for Pt.

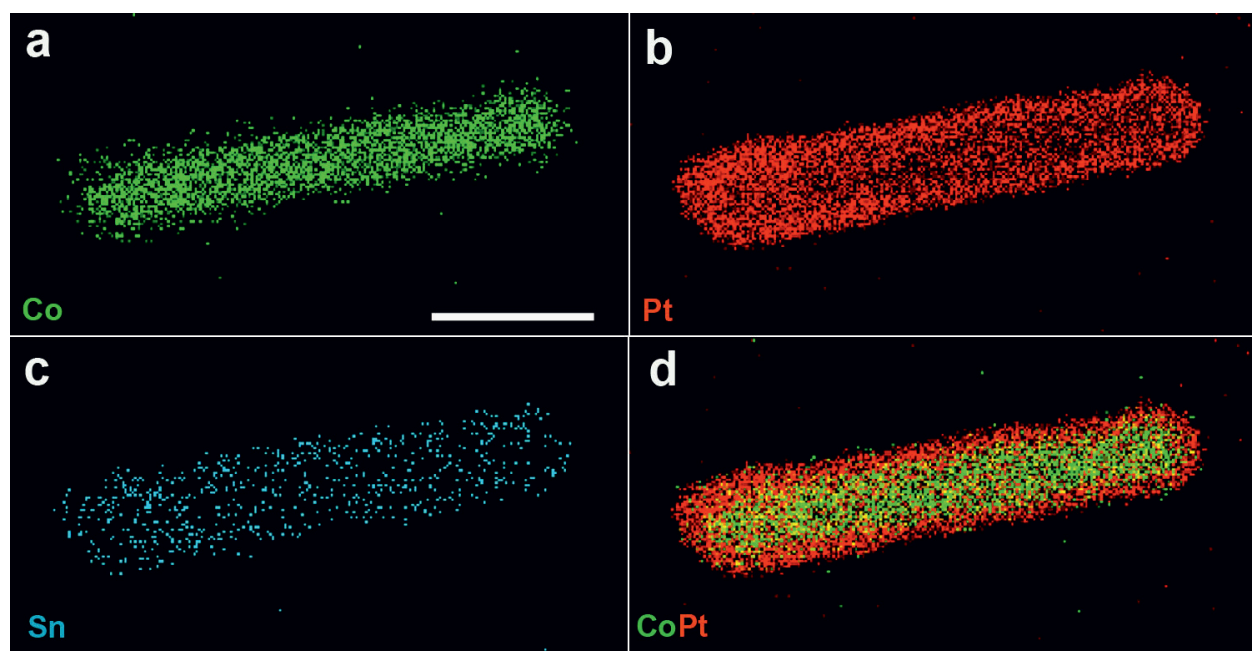

Figure 4. STEM- EDX maps showing the distribution of each element separately (a-c), as well as of the couple CoPt on an isolated nanorod (d), which has a completely covered Co core (Co: green, Pt: red, Sn: blue). Scale bar for all EDX maps is $10 \mathrm{~nm}$.

The STEM-EDX measurements performed on fully covered isolated nanorods (Figure 4a-d) reveal that a shell in which both Sn and Pt are present has smoothly grown on Co. Again, the presence of Sn does not appear to be strictly limited to a layer between Co and the noble metal.

The resistance of the Co@SnAu and Co@SnPt nanorods to oxidation was investigated by TEM observations after exposure to air and distilled water for 2 days (Supporting Figure S6). Water affects the Au- and Pt-coated nanorods differently. Almost all Co@SnAu nanorods were severely attacked, but fully coated Co@SnPt specimens are seemingly unaffected by water. Nevertheless, incompletely covered or naked Co@SnPt rods appear corroded. From the TEM/HRTEM/HAADF images of the sample prior to water exposure, all Co@SnAu nanorods 
(Figure 1and Figure S3) appear to be coated in a similar manner. However, important variations of the shell thickness along their length can be observed, and at some points the shell is very thin (2 or 3 atomic layers). We presume that thinner Au shell areas are more reactive and prone to etching. The Pt shell (Figure 3) is much thicker and smoother, presenting fewer shell thickness variations. The fully covered Co@SnPt nanorods remain intact after water exposure, but incompletely covered or naked nanorods are corroded.

These results prompted us to use Pt and Au successively. The idea was to use these two metals of limited miscibility, ${ }^{52}$ in order to avoid extended overgrowth of the second noble metal on areas already occupied by the first metal, thus favoring deposition on the uncovered Co surface. In Figure 5 we present the core-shell nanorods prepared by three successive steps: (i) [ $\left.\left\{\mathrm{Sn}\left(\mathrm{NMe}_{2}\right)_{2}\right\}_{2}\right]$ treatment of the Co-NRs, (ii) 3 cycles of Pt deposition and (iii) 2 cycles of Au deposition (See Supporting Information). A TEM image of the Co@SnPtAu nanorods is shown in (Figure 5a). A less smooth surface in comparison to the Co@SnAu and Co@SnPt nanorods is observed. The HRTEM image presented in Figure 5b shows the presence of a polycrystalline shell, whereas STEM-EDX measurements (Figure 5c-f) reveal that indeed, Pt and Au are complementary as shown further in Supporting Figure S7. It is important to point out that Co@SnPtAu nanorods do not seem to be affected by water, as indicated by the TEM inspection, which shows that after 2 days of water exposure, the nanorods are apparently intact (Supporting Figure S8). 


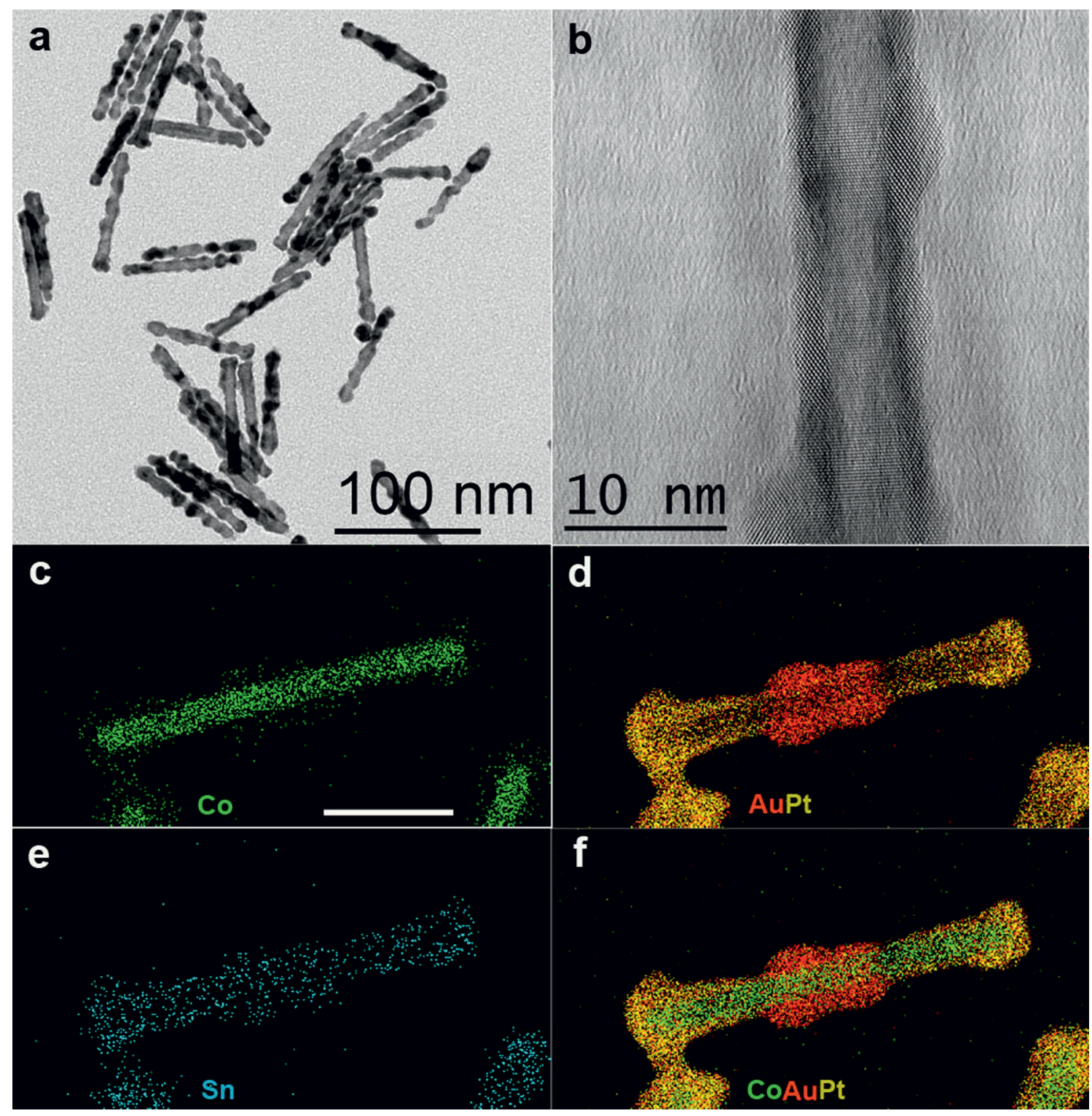

Figure 5. (a) Low magnification TEM image of a Co@SnPtAu nanorod sample (scale bar: 100 $\mathrm{nm})$. (b) HRTEM image on an isolated nanorod, revealing the polycrystalline nature of the shell (scale bar: $10 \mathrm{~nm}$ ). (c-f) STEM- EDX maps showing the location of the elements on a fully covered nanorod (c: Co, d: Au and Pt, e: Sn, f: Co, Au and Pt). (Co: green, Sn: blue, Au: red Pt: yellow). Scale bar for all EDX maps is $20 \mathrm{~nm}$.

An inversion of the noble metal addition order has also been attempted. The deposition of Au prevents Pt deposition. This result is not unexpected, considering that: (i) Au does not let any 
uncovered areas in order for Pt to be deposited and (ii) Pt and Au do not occupy the same area. The TEM micrograph of this sample and EDX maps of a single nanorod are shown in Supporting Figure S9).

X-Ray Diffraction (XRD) measurements on the as prepared core-shell powders are dominated by the signals of Au or/and Pt as shown in the XRD patterns presented in Supporting Figure S10. Thus, no information can be obtained from XRD about the state of tin in the core-shell nanorods. In order to better understand the structure of the core-shell nano-objects, X-Ray Absorption Spectroscopy (XAS) measurements were performed on as prepared Co@SnM (M= Au and/or Pt) samples, and the results are presented in Figure 6. The Co K-edge X-ray Absorption Near Edge Structure (XANES) spectra for all samples, and the reference are presented in Figure 6a. Together with the Supporting Table S1 these results show that in all samples Co is metallic. The corresponding XANES spectra for the Sn, Au and Pt edges are presented in Supporting Tables S2-S4 and Supporting Figs. S11-S13. This investigation revealed that all samples contain only zero-valent Co, Pt and Au. However, Sn was metallic in Co@SnPt and Co@SnPtAu, but nonmetallic in Co@SnAu. The coordination numbers and bond distances were determined from the Extended X-Ray Absorption Fine Structure (EXAFS) data, allowing identification of alloying where present. Selected plots of the Fourier-transformed EXAFS data are shown in Figure 6b-d and in Table 1 and Supporting Figs. S14-S16. No evidence for alloy formation between Co or $\mathrm{Au}$ with any other element was detected in any sample, and in addition, no Pt-Au alloys were observed in Co@SnPtAu. However, at the Sn K-edge, Sn-Pt scattering was observed for both Co@SnPt and Co@SnPtAu suggesting formation of Pt-Sn alloys in both samples. At the Pt $\mathrm{L}_{3^{-}}$ edge, Pt-Sn scattering could not be detected in either Co@SnPt or Co@SnPtAu, likely due to the much larger number of Pt-Pt scatters which obscures the signal from the Pt-Sn scattering. The 
lack of detectable Sn-Sn scattering suggests that there were no large Sn-rich regions in the shell. Interestingly, for the Co@SnAu sample there was only scattering characteristic of Sn bonded to 4 light scatterers (O or N) (Supporting Figure S15), in agreement with the observation from the XANES that the $S n$ is non-metallic and hence unalloyed.
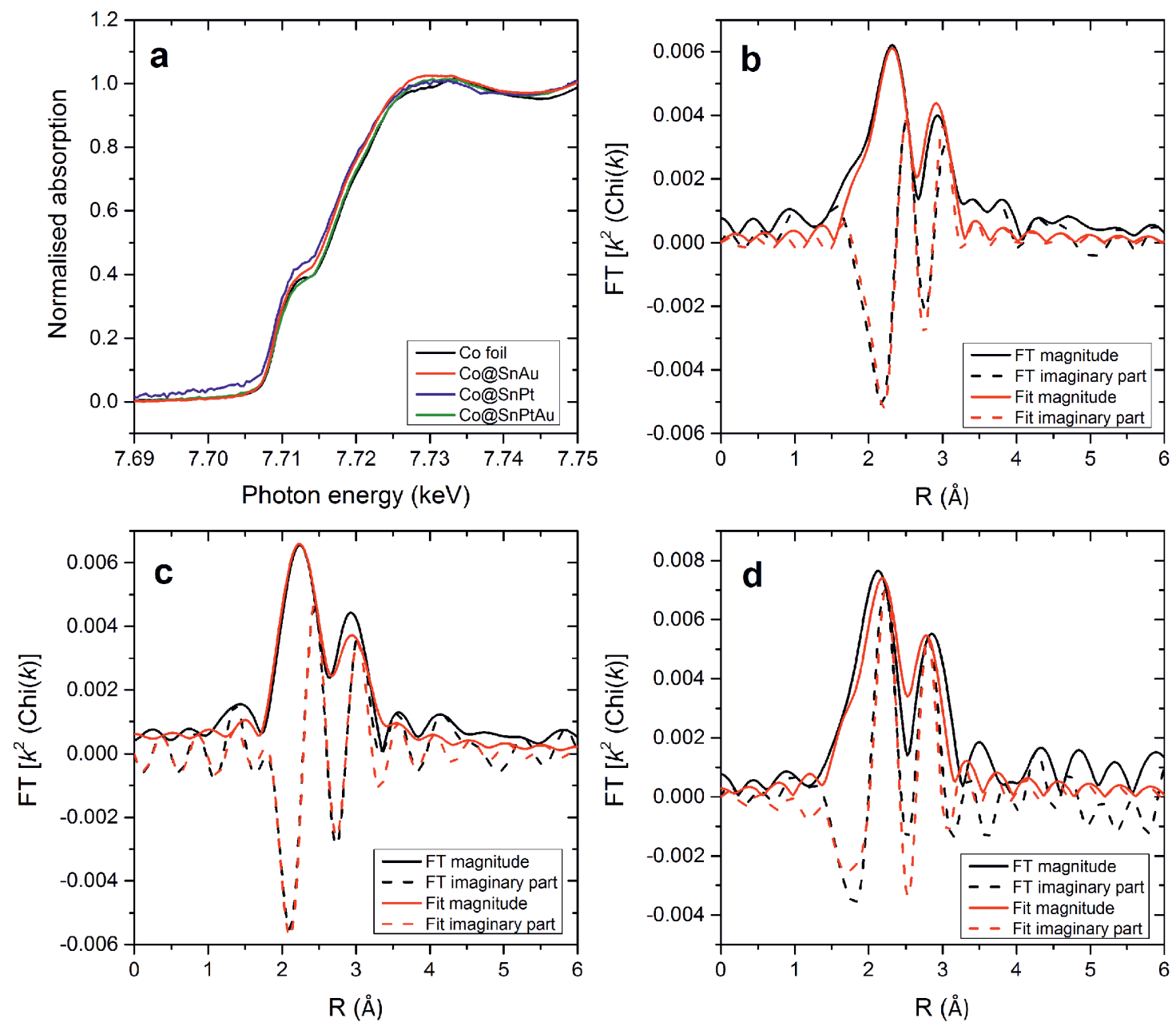

Figure 6. (a) XANES Co K-edge spectra of as prepared samples obtained under inert conditions and Co-foil reference, demonstrating that Co is metallic. (b) Au L3-edge R space EXAFS fitting result of Co@SnAu. k2: $\Delta \mathrm{k}=2.610 .5 \AA 1$, evidencing that Au is metallic and not alloyed. The EXAFS of Co@SnPtAu was similar. (c) Pt L3-edge R space EXAFS fitting result of Co@SnPt. k2: $\Delta \mathrm{k}=2.79 .2 \mathrm{~A} 1$, showing that Pt is metallic. The EXAFS of Co@SnPtAu was 
similar. (d) Sn K-edge R space EXAFS fitting result of Co@SnPt. k2: $\Delta \mathrm{k}=2.710 .2 \AA 1$. The EXAFS at the Sn edge does not correspond to Sn metal and instead is indicative of SnPt scattering only, suggesting formation of a PtSn alloy. Co@SnPtAu was similar, also indicating PtSn alloy formation (see Figure S16), whereas Co@SnAu contained nonmetallic and hence nonalloyed Sn species (see Figure S15).

Table 1. Fitted EXAFS data of the samples and references at all edges

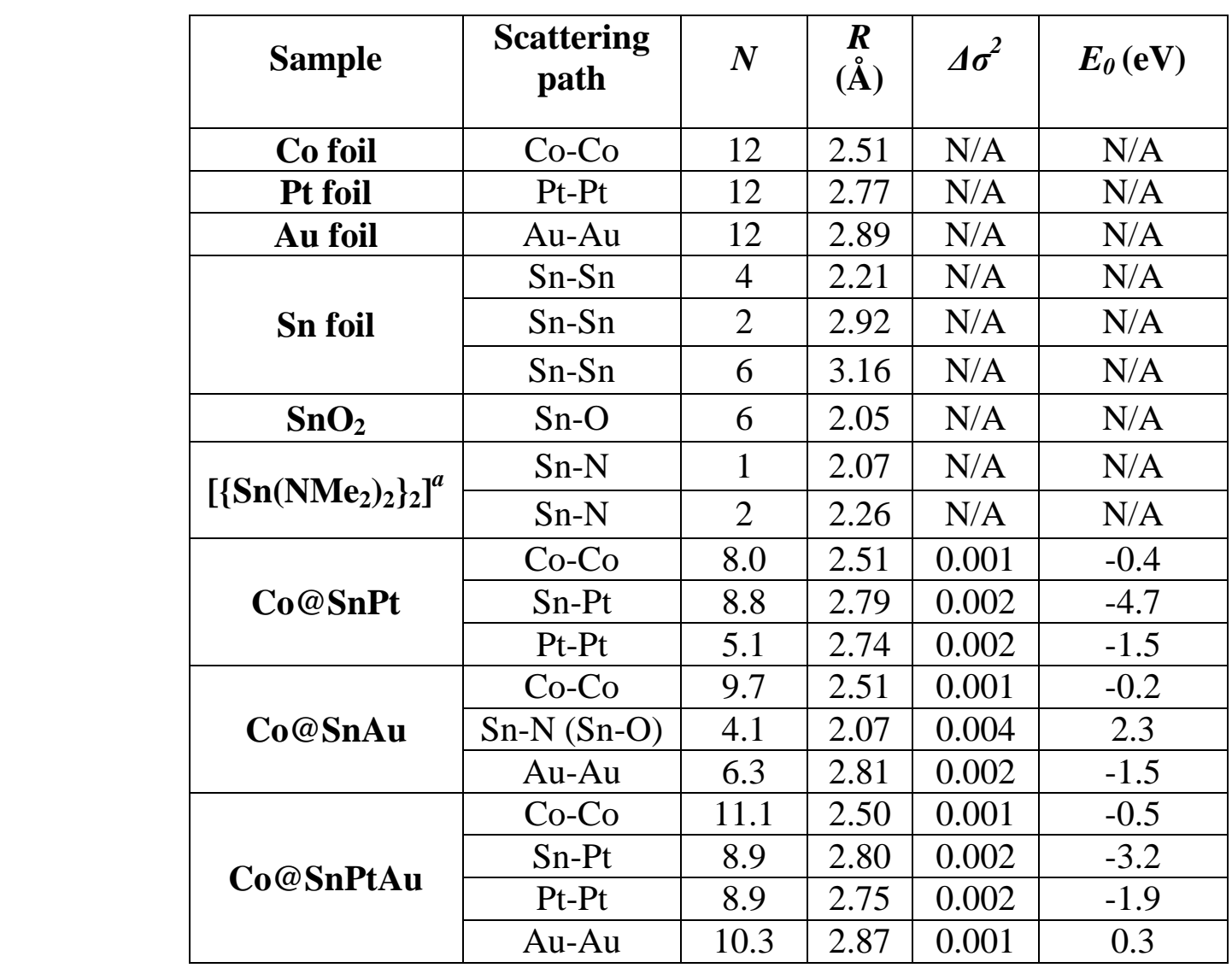

${ }^{a}$ Reference 53

Sn is the crucial factor that enables the noble metal shell development. Thus, in a first approach we used TEM to examine the Co-NRs after treatment with [ $\left.\left\{\mathrm{Sn}\left(\mathrm{NMe}_{2}\right)_{2}\right\}_{2}\right]$ and washing with toluene. As in the case of the Co@SnM nanorods, only STEM-EDX analysis 
shows the presence of Sn on Co-NRs (Supporting Figure S17). X-ray Photoelectron

Spectroscopy (XPS) measurements on Co@Sn after washing confirm the metallic nature of Co. ${ }^{54}$ Furthermore, these measurements show that part of the tin is $\mathrm{Sn}^{0}$ but the major part is $\mathrm{Sn}^{2+}$ associated to $\mathrm{O}$, which presumably originates from the Co-seed native laurate ligands $\left(\mathrm{Sn}^{2+} / \mathrm{Sn}^{0}=\right.$ 3.2). The presence of a small percentage of Sn-N bonds cannot be excluded. Since no $\mathrm{H}_{2}$ has been used during treatment with $\left[\left\{\mathrm{Sn}\left(\mathrm{NMe}_{2}\right)_{2}\right\}_{2}\right]$, and HDA is not able to reduce $\left[\left\{\mathrm{Sn}\left(\mathrm{NMe}_{2}\right)_{2}\right\}_{2}\right]$ at room temperature, the presence of metallic Sn is probably due to a limited galvanic displacement reaction between $\mathrm{Co}(0)$ and $\mathrm{Sn}(\mathrm{II})$ at the $\mathrm{Co} / \mathrm{Sn}$ interface (for XPS details see Supporting Figure S18 and Supporting Table S5).
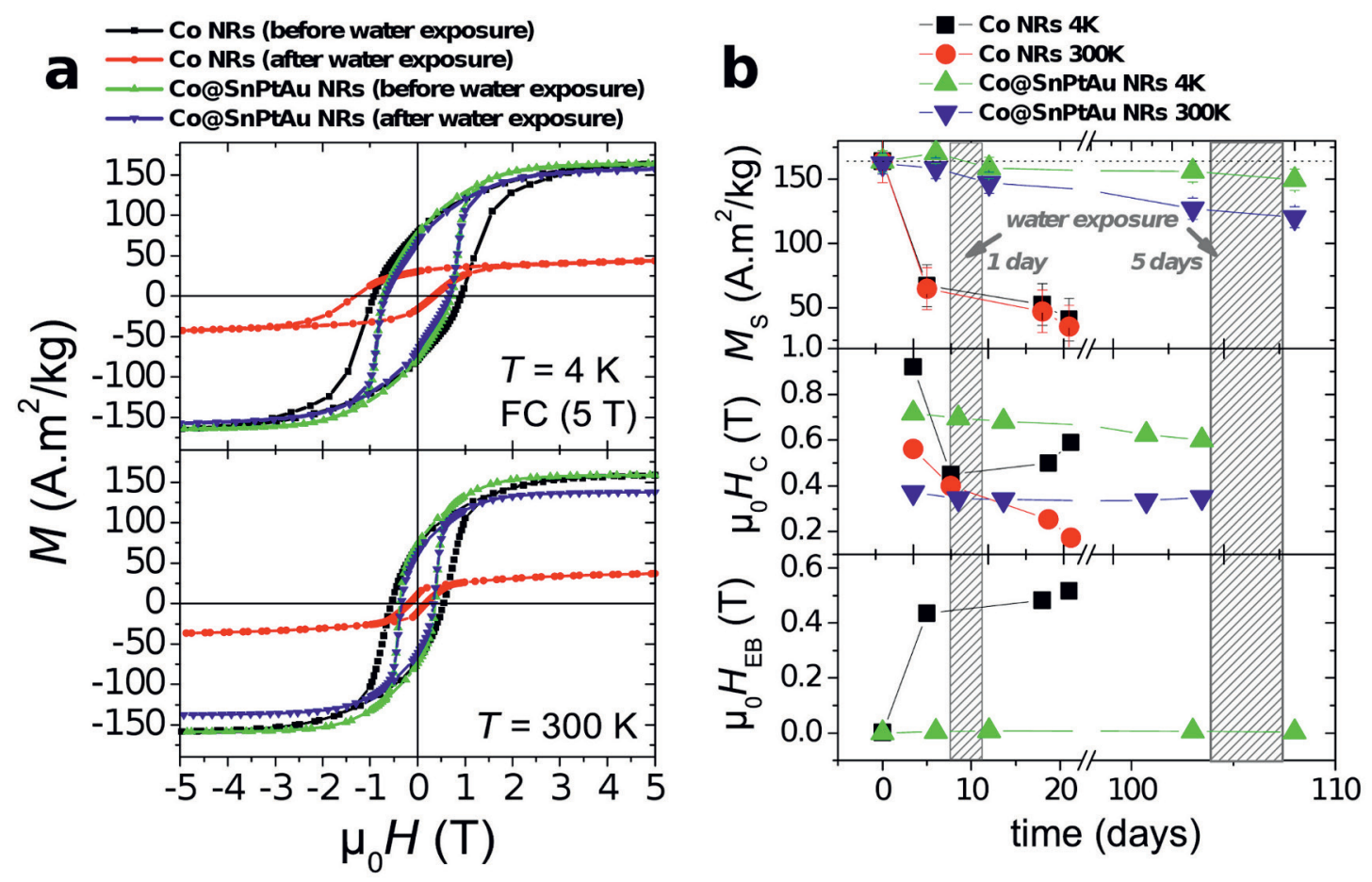

Figure 7. (a) Hysteresis loops measured at $\mathrm{T}=4 \mathrm{~K}$, and at $\mathrm{T}=300 \mathrm{~K}$ after FC under $5 \mathrm{~T}$ of Co(black squares: before air- and water-exposure; red squares: after 5 days air-exposure and 1 day water-exposure) and Co@SnPtAu nanorods (green triangles: before air- and water-exposure; blue triangles: after 6 days air-exposure and 1 day water-exposure). (b) Time evolution of the 
saturation magnetization (Ms) (top), coercive field $(\mu 0 \mathrm{Hc})$ (middle), and exchange bias field ( $\mu$ HEB (bottom). The Co-NRs traces at $4 \mathrm{~K}$ correspond to the black squares, and at $300 \mathrm{~K}$ to the red circles. Co@SnPtAu nanorod traces at 4 K correspond to the green triangles, and at 300 $\mathrm{K}$ to the blue triangles. The shaded areas indicate the time interval during which the nanorods have stayed in contact with water ( 1 and 5 days). During the time intervals between the two water exposure treatments the samples were exposed to air. All water and air exposure treatments have been performed at room temperature.

Thus, Sn treatment seems to influence two parameters that dominate the reaction outcome. [ $\left.\left\{\mathrm{Sn}\left(\mathrm{NMe}_{2}\right)_{2}\right\}_{2}\right]$ acts as a metal-containing ligand that "wets" the entire Co surface, presumably by displacing, or reacting with, existing ligands of the Co surface. This effect could account for its positive role on the conformal shell formation independently of the shell-metal used. Additionally, Sn affects surface/interfacial energies allowing the formation of a favorable interface even between immiscible metals ( $\mathrm{Co}-\mathrm{Au})$. Whereas the exact nature of the Sn species present on Co@Sn intermediate is elusive, it is possible that upon contact with Co-NRs, a small amount of the [ $\left.\left\{\mathrm{Sn}\left(\mathrm{NMe}_{2}\right)_{2}\right\}_{2}\right]$, not detectable by XAS in the final core-shell nanorods, but visible by XPS in the Co@Sn sample, is reduced in a transmetallation reaction, but most of the Sn is non-metallic. Sn species can play a role similar to the role of "surfactants" in the epitaxial thin film growth. ${ }^{43}$ The EDX map (Fig. 2), that does not exclude non-metallic Sn associated to lighter scatterers "floating" to the surface, indicate that such a function is possible. Subsequent reaction with [PtMe $2 \mathrm{COD}$ ] under $\mathrm{H}_{2}$ reduces both $\mathrm{Pt}$ and $\mathrm{Sn}$, which form a continuous shell in which $\mathrm{Sn}$ is present as an alloy. Subsequent $\left[\mathrm{AuClPPh}_{3}\right]$ reduction takes place mainly on $\mathrm{Pt}$ deficient areas. On the other hand, Sn is practically non-metallic in Co@SnAu. This difference 
could be due to the Pt acting as an efficient hydrogenation catalyst in the cases of Co@SnPt and Co@SnPtAu.

Whereas the above experiments give an indication about the coating efficiency of the SnPtAu shell, the sine qua non condition for nanorod implementation in an aqueous environment is the conservation of the Co-core magnetic properties. Thus, magnetic measurements were performed both at $300 \mathrm{~K}$, as well as at $4 \mathrm{~K}$, on the water-resistant Co@SnPtAu, before and after exposure of the sample (i) to air, and (ii) to water (Figure 7). The air- and water-exposure treatments were performed at room temperature. For comparison, Co-NRs were also measured following the same experimental protocol. The initial magnetization is measured for both Co-NRs and Co@SnPtAu, freshly synthesized samples, kept in the glove box. These measurements represent the reference of the air/water-stability of the nanorods and are referred as " $t=0$ ” (see Figure 7). Zero Field Cooled (ZFC) and Field Cooled (FC) at 5T measurements are realized at $4 \mathrm{~K}$ in order to confirm the metallic state of the Co before exposure to air or water. After measuring the magnetic properties at $\mathrm{t}=0$, the Vibrating Sample Magnetometry (VSM) capsules were opened to the air. After measurement of the effect of air exposure, they were completely filled with deionized water (around $40 \mu \mathrm{L}$ ). After the complete evaporation of the water, ZFC-FC measurements were performed again. We emphasize that only this protocol that measures the same samples before and after exposure allows a quantitative analysis and control of the variation of the magnetization of the sample after exposure. In Table 2 we present the evolution of the magnetic measurements of bare Co and Co@SnPtAu nanorods upon air and water exposure. 
Table 2. Values of $M s, \mu_{0} H c$ and $\mu_{0} H_{\mathrm{EB}}$ of the Co and Co@SnPtAu nanorods before and after air- and water-exposure.

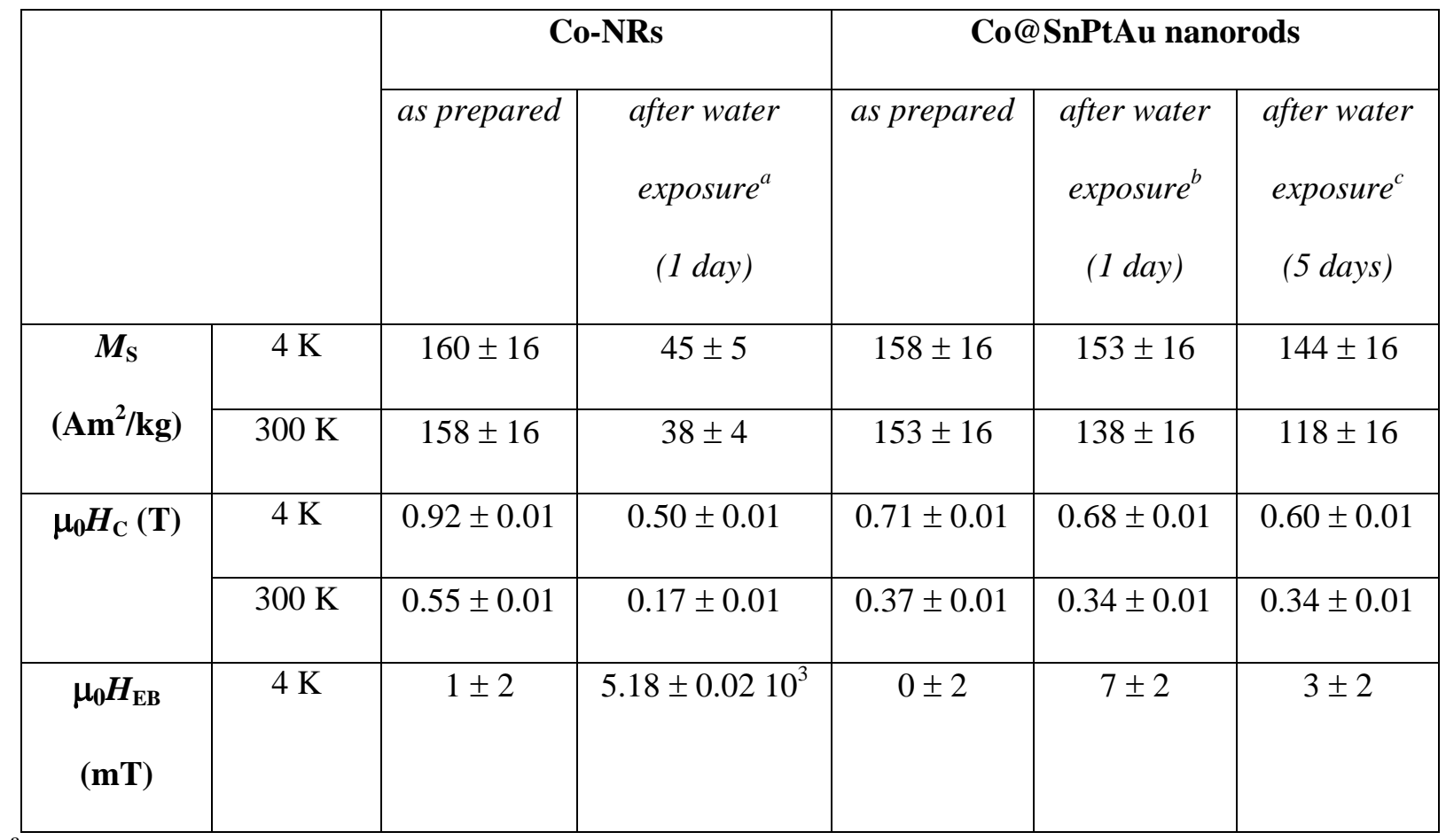

a the water exposure is preceded by 5 days air exposure.

${ }^{\mathrm{b}}$ the water exposure is preceded by 6 days air exposure.

${ }^{\mathrm{c}}$ the water exposure is preceded by 6 days air exposure 1 day in water exposure and 3 months of additional air exposure. 


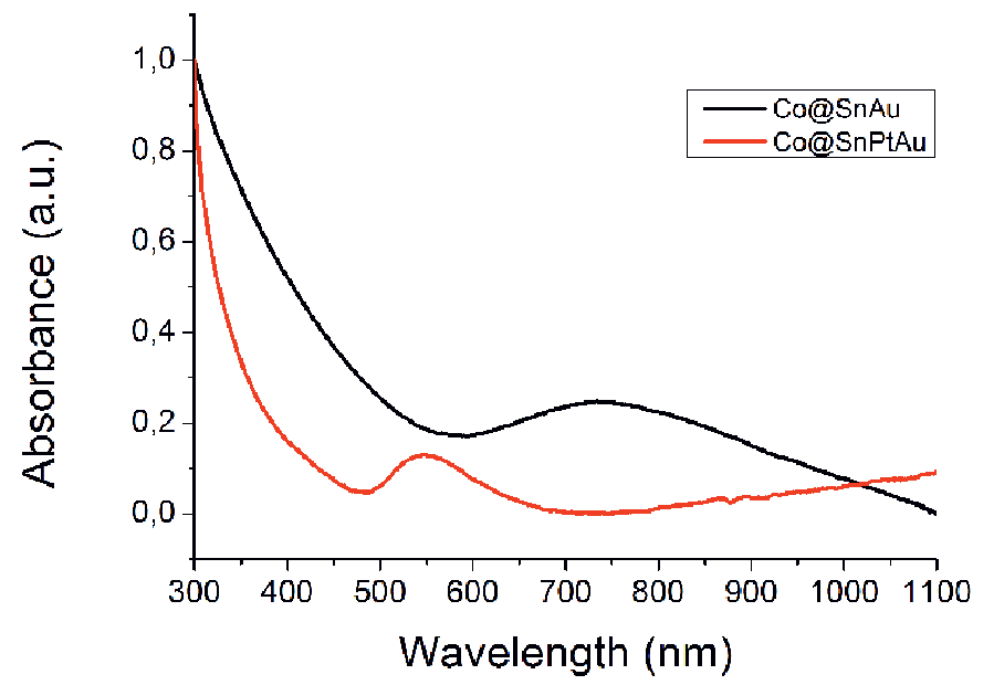

Figure 8. UV-vis absorbance spectra of toluene suspension of Co@SnAu (black trace) and Co@SnPtAu nanorod (red trace).

Before any exposure to air or water, both samples present a magnetization at saturation very close to the expected bulk value of $163 \mathrm{Am}^{2} / \mathrm{kg}\left(M_{\mathrm{S}}=160 \mathrm{Am}^{2} / \mathrm{kg}\right.$ and $158 \mathrm{Am}^{2} / \mathrm{kg}$ at $T=4 \mathrm{~K}$ for Co-NRs and Co@SnPtAu respectively). The absence of exchange bias (EB) after field cooling (FC) under a 5T magnetic field (see Figure 7a and Table 2) corroborates the metallic character of the Co core, since the formation of $\mathrm{CoO}$ would lead to an exchange bias due to antiferromagnetic coupling. ${ }^{55}$ These results also show that coating does not affect the $M_{\mathrm{S}}$. Moreover, the high coercive field $\left(\mu_{0} H_{\mathrm{C}}=0.71 \mathrm{~T}\right.$ at $4 \mathrm{~K}$ ), of the Co@SnPtAu nanorods (just below the one of CoNRs), ${ }^{11}$ confirms that their effective magnetic anisotropy is high enough $\left(>4 * 10^{5} \mathrm{~J} / \mathrm{m}^{3}\right)$ for applications in which hard magnetic materials are required. As expected, the Co-NRs present several obvious signs of oxidation after 5 days exposure to the air: a large drop of $M_{\mathrm{S}}$ and of $\mu_{0} H_{\mathrm{C}}$, and a high exchange bias field $\mu_{0} H_{\mathrm{EB}} \sim 0.5 \mathrm{~T}\left(H_{\mathrm{EB}}=\left(H_{\mathrm{C}^{+}}-H_{\mathrm{C}-}\right) / 2\right.$ with $H_{\mathrm{C}^{+}}$and $H_{\mathrm{C}^{-}}$the positive and negative coercive field respectively) (see Figure 7b). 
On the contrary, the magnetization of Co@SnPtAu nanorods does not seem to be sensitive to air exposure after 6 days. The samples exposed to air were then immersed into distilled water for 1 day. Whereas the oxidation unambiguously advanced in the case of Co-NRs, the Co@SnPtAu nanorods only displayed slight changes in their magnetic properties. When measured at $T=300$ $\mathrm{K}$, their magnetization was still high but decreased from $M_{\mathrm{S}}=153 \mathrm{Am}^{2} / \mathrm{kg}$ (before water exposure) to $M_{\mathrm{S}}=138 \mathrm{Am}^{2} / \mathrm{kg}$. Interestingly, the magnetization decrease was less pronounced at $4 \mathrm{~K}\left(M_{\mathrm{S}}=153 \mathrm{Am}^{2} / \mathrm{kg}\right)$, at which $97 \%$ of the initial $M_{\mathrm{S}}$ value, along with a small exchange bias field $\mu_{0} H_{\mathrm{EB}}=7 \mathrm{mT}$ were measured. The Co@SnPtAu nanorods were then subjected to an extended conditioning period, first to the air for 3 months, and subsequently to water for 5 days, giving rise to a monotonous decrease of $M_{\mathrm{S}}$, which corresponds to $91 \%$ and $74 \%$ of the initial $M_{\mathrm{S}}$ value measured at $4 \mathrm{~K}$ and $300 \mathrm{~K}$ respectively. However this decrease was much less severe than in the case of Co-NRs, for which $24 \%$ and $21 \%$ of the initial $M_{\mathrm{S}}$ value were measured at $4 \mathrm{~K}$ and $300 \mathrm{~K}$ respectively. This very limited oxidation could be due to the fact that grain boundaries between Pt and Au crystallites on the shell are more reactive sites, from which corrosion takes place upon extended water exposure. It is also possible, that some imperfectly covered areas are still remaining. Despite the drop of 26\% of $M_{\mathrm{S}}$ at $300 \mathrm{~K}$, the Co@SnPtAu nanorods still present a high $M_{\mathrm{S}}\left(\sim 115 \mathrm{Am}^{2} / \mathrm{kg}\right)$ and a coercive field large enough $(0.34 \mathrm{~T}$ at $300 \mathrm{~K})$ for applications requiring both high $M_{\mathrm{S}}$ and a hard magnetic behavior. To our knowledge, air- or water-resistant magnetic nano-objects of both high Ms and anisotropic shape have not been reported so far. Indeed, existing ferromagnetic nanorods, which can be used in water, have much lower Ms than Co@SnPtAu nanorods. ${ }^{56-58}$ On the other hand, the existing nano-objects of high Ms are either not resistant towards oxidation or isotropically shaped. ${ }^{14,15,59}$ 
Finally, studies of the optical properties of core-shell nanorods have been performed for toluene dispersions of core-shell nanorods. In Figure 8, we present UV-Vis normalized absorbance spectra of Co@SnAu and Co@SnPtAu nanorods. The homogeneously coated Co@SnAu nanorods show a local absorbance maximum at about 730 nm, which we attribute to a longitudinal localized surface plasmon resonance (LSPR) mode in the tin-containing gold shell. For the Co@SnPtAu nanorods, a local absorbance maximum is obtained at about 540 nm, which we assign to LSPR modes in small gold patches on the surface of the Pt/Au domain-like shell structure.

\section{CONCLUSION}

We have achieved the synthesis of air and water-resistant highly magnetic and ferromagnetic nanorods through treatment of Co-NRs with $\left[\left\{\mathrm{Sn}\left(\mathrm{NMe}_{2}\right)_{2}\right\}_{2}\right]$. This strategy tackles two important problems connected to the growth of a complete shell of a different material on an anisotropic nano-object, that is, homogeneous “wetting” of all Co nanorod facets despite their different reactivity, and the formation of an energetically favorable continuous interface between dissimilar materials. Thus, Sn treatment has enabled the subsequent development of a conformal noble metal shell, which renders them air- and water-resistant. The metallic character and the magnetic properties of the cobalt core are preserved even after extended periods of exposure to ambient atmospheric conditions as well as to water. This work opens the perspective of implementation of the Co@SnPtAu nano-objects in the domain of in vitro immunodiagnostics. Last but not least, although the present work is mainly focused on cobalt nanorods, this new chemical approach has a broad potential, and can be adapted to the synthesis of core-shell 
nanoparticles of different shapes and compositions with applications spanning from nanomedicine to ferrofluids and catalysis.

\section{METHODS}

Due to the air sensitivity of the metal precursors, as well as of the Co-NRs, all experiments were performed under inert conditions, in the interior of an Ar glove-box or by using standard Schlenk techniques. The starting Co-NRs have been prepared by slight modifications of an already published procedure. ${ }^{10}$ The naked Co-NRs were treated with $\left[\left\{\mathrm{Sn}\left(\mathrm{NMe}_{2}\right)_{2}\right\}_{2}\right]$ before coating by the noble metals takes place. A detailed description of the standard procedures is given in the Supporting Information.

The dimensions of the samples presented in Figures 1-5 are given below. It has to be noted that all core@shell nanorods are denoted as Co@SnX. The shell is symbolized according to the order by which the metal precursors have been deposited on the Co core. It also reflects the presence of various elements on the shell as a whole, but does not automatically indicate alloy formation.

Co@SnAu: Sample shown in Figures 1 and 2. Before coating: length $(\mathrm{L})=61 \pm 6$ nm, diameter (d) $=5.7 \pm 0.7 \mathrm{~nm}$ (Supplementary Figure S19 a); after coating: $\mathrm{L}=61 \pm 5 \mathrm{~nm}, \mathrm{~d}=7.2 \pm 0.7 \mathrm{~nm}$ (Supplementary Figure S19 b). EDX analysis on several areas of a TEM grid yields the following mean values $\%$ at: $\mathrm{Co}=67 \%, \mathrm{Sn}=10 \%, \mathrm{Au}=23 \%$.

Co@SnPt: The size distribution taking into account only the fully covered nanorods indicates that the shell thickness is about $1 \mathrm{~nm}$. Sample shown in Figure 3 and 4: Before coating: $\mathrm{L}=44 \pm 4$ $\mathrm{nm}, \mathrm{d}=5.7 \pm 0.5 \mathrm{~nm}$ (Supplementary Figure S19 c); after coating: $\mathrm{L}=46 \pm 3 \mathrm{~nm}, \mathrm{~d}=7.7 \pm 0.6 \mathrm{~nm}$ (Supplementary Figure S19 d). 
EDX analysis on several areas of a TEM grid yields the following mean values $\%$ at: $\mathrm{Co}=$ $52 \%, \mathrm{Sn}=6 \%, \mathrm{Pt}=42 \%$.

Co@SnPtAu: The mean length and diameter of the final nanorods have increased, however the irregular metal deposition does not allow a reliable and statistically relevant diameter measurement Sample shown in Figure 5: naked nanorods: $\mathrm{L}=74 \pm 5 \mathrm{~nm}, \mathrm{~d}=5.9 \pm 0.5 \mathrm{~nm}$; coreshell nanorods (Supplementary Figure S19 e): L $=75 \pm 6$ nm, d: around 9 nm (Supplementary Figure S19 f). EDX analysis on several areas of a TEM grid yields the following mean values \% at: $\mathrm{Co}=35 \%, \mathrm{Sn}=4 \%, \mathrm{Pt}=40 \% \mathrm{Au}=21 \%$.

\section{TEM-HRTEM}

Samples for TEM observations were prepared in a glove box, by drop-casting toluene suspensions of the nanorods on a carbon coated copper grid.

TEM characterization was performed on a JEOL JEM 1011 microscope, equipped with a W thermionic electron source and a Mega-View Olympus CCD camera and working at an acceleration voltage of $100 \mathrm{kV}$, or with a JEOL JEM 1400 microscope equipped with LaB6 thermionic electron source and working at an acceleration voltage of $120 \mathrm{kV}$. HRTEM observations were realized using a $200 \mathrm{kV}$ FEI Tecnai microscope equipped with a field emission gun and a Cs corrector which avoids delocalization effects at interfaces (point resolution of $0.12 \mathrm{~nm}$ ). The structural features of the nanostructures imaged by HREM were studied by means of 2D Fourier analysis.

\section{STEM-EDX}


STEM-EDX measurements were performed using an aberration-corrected cubed FEI-Titan 60300 electron microscope operated at $200 \mathrm{kV}$. For the acquisition of the maps, a ChemiSTEM system was used. ${ }^{60}$ For the acquisition and quantification of the maps, the ESPRIT software was used.

Routine EDX was performed using JEOL JEM 2100F electron microscope operated at $200 \mathrm{kV}$ equipped with a field emission gun (FEG) and SDD Brucker (light elements detection), with a resolution of $127 \mathrm{eV}$.

\section{XRD}

Powder XRD data were obtained on a Panalytical Empyrean instrument with samples enclosed

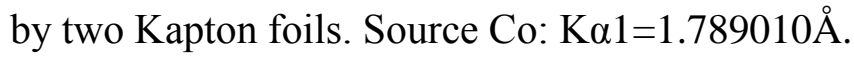

\section{XAS}

X-ray absorption measurements were acquired at the Co K-edge (7.709 keV), Sn K-edge (29.200 keV), Pt L-edge (11.564 keV) and Au L3-edge (11.919 keV) on the bending magnet beam line of the Materials Research Collaborative Access Team (MRCAT) at the Advanced Photon Source, Argonne National Laboratory. The data were collected in transmission step-scan mode. Photon energies were selected using a water-cooled, double-crystal Si (111) monochromator, which was detuned by approximately $50 \%$ to reduce harmonic reflections. The ionization chambers were optimized for the maximum current with linear response $\left(\sim 10^{10}\right.$ photons detected/sec) with $10 \%$ absorption in the incident ion chamber and $70 \%$ absorption in the transmission X-ray detector. The appropriate metal foil spectrum was acquired simultaneously with each sample measurement for energy calibration. 
Nanorod samples after being washed and dried were pressed into a cylindrical sample holder consisting of six wells, forming a self-supporting wafer. All samples were prepared in a $\mathrm{N}_{2}$ glove box and the sample holder was placed in a quartz tube (1-in. OD, 10-in. length) sealed with Kapton windows by two Ultra-Torr fittings. Samples were pressed directly as received to achieve an absorbance $(\mu \mathrm{x})$ of approximately 1.0 .

Standard data reduction techniques were employed to fit the data using the WINXAS 3.1 software program. ${ }^{61}$ The normalized, energy-calibrated absorption spectra were obtained using standard methods. The edge energy was determined from the maximum of the first peak of the first derivative of the XANES spectrum. For the Co, Pt, Au edges experimental phase shift and backscattering amplitudes were measured using the appropriate metal foils (coordination numbers and bond distances shown in Table 1). For the $\mathrm{Sn}$ edge, $\mathrm{SnO}_{2}$ was used as the reference for Sn bonded to a light scatterer (Sn-O or Sn-N scattering). The EXAFS and XANES of the $\left[\left\{\mathrm{Sn}\left(\mathrm{NMe}_{2}\right)_{2}\right\}_{2}\right]$ precursor was also obtained for comparison. Since experimental references were not available for alloys, theoretical phase and amplitude functions for Sn-Co and Sn-Pt were calculated with FEFF6 ${ }^{62}$ using a two atom calculation, calibrated to the appropriate foil. The EXAFS parameters were obtained by a least square fit in $R$-space of the $k^{2}$-weighted Fourier Transform (FT) data for all samples except the Sn K-edge of sample Co@SnAu where the EXAFS parameters were obtained by a least square fit in $k$-space of the $k^{2}$-weighted BackFourier Transform data. The Debye-Waller factor $\left(\Delta \sigma^{2}\right)$ is dependent on the amount of disorder in the sample and was fixed at a given edge at either 0.001 or 0.002 for metallic particles or 0.004 for light scatterers. All fits were single shell.

\section{XPS}


XPS measurements were carried out with a Thermo Scientific K-Alpha X-ray photoelectron spectrometer, using a focused monochromatized $\mathrm{Al} \mathrm{K} \alpha$ radiation $(\mathrm{h} v=1486.6 \mathrm{eV})$. The XPS spectrometer was directly connected through a glove box under argon atmosphere, in order to avoid moisture/air exposure of the samples. For the Ag 3d5/2 line the full width at halfmaximum (FWHM) was $0.50 \mathrm{eV}$ under the recording conditions. The X-ray spot size was 400 $\mu \mathrm{m}$. Peaks were recorded with constant pass energy of $20 \mathrm{eV}$. The pressure in the analysis chamber was less than $2 \times 10^{-8} \mathrm{~Pa}$. Short acquisition time spectra were recorded at the beginning and at the end of each experiment to check that the samples did not suffer from degradation during the measurements. Peak assignments were made with respect to reference compounds analyzed in the same conditions. The binding energy scale was calibrated from the hydrocarbon contamination using the $\mathrm{C} 1$ s peak at $285.0 \mathrm{eV}$. Core peaks were analyzed using a nonlinear Shirley-type background. ${ }^{63}$ The peak positions and areas were optimized by a weighted leastsquares fitting method using 70\% Gaussian, 30\% Lorentzian line shapes. Quantification was performed on the basis of Scofield's relative sensitivity factors. ${ }^{64}$ Several XPS analyses were performed at different positions to make the results statistically reliable.

\section{Magnetic measurements}

The magnetic properties of Co and Co@SnPtAu nanorods have been characterized by VSM using a Quantum Design PPMS 9T EverCool II.

In order to ensure a reliable quantitative analysis of the magnetization evolution upon air and water exposure, the same sample was used throughout the whole series of measurements, thus avoiding any errors stemming from the use of different samples. Standard VSM capsules were filled with a known amount (a few mg) of sample and sealed, without any other material 
commonly used to freeze the magnetic compound (vacuum grease, epoxy...). The addition of the blocking material (vacuum grease, epoxy...) would prevent contact of the nanorods with air and water. To avoid any oxidation, the samples are prepared in the glove-box, and transferred to the cryostat in a Schlenck vessel. The VSM capsule containing the sample is rapidly introduced into the VSM in order to avoid exposure of the sample to the air. ZFC was performed by cooling the sample down to $4 \mathrm{~K}$ without application of magnetic field and field-dependent magnetization was recorded at $4 \mathrm{~K}$. The temperature was then increased to $300 \mathrm{~K}$, and a $5 \mathrm{~T}$ magnetic field was applied. A second field- dependent magnetization was recorded after having cooled down to 4K while the 5T magnetic field was applied. The mass magnetization of Co is calculated by taking into account the metallic fraction of Co obtained by ICP.

\section{Absorbance measurements}

Absorbance measurements were carried out with a Perkin-Elmer Lamda35 UV-Vis spectrophotometer by dispersing the nanorods in toluene. Measurements were carried out at room temperature.

\section{ASSOCIATED CONTENT}

Supporting Information. Details on nanorod synthesis, TEM of Au and Pt growth on Co-NRs without [ $\left.\left\{\mathrm{Sn}\left(\mathrm{NMe}_{2}\right)_{2}\right\}_{2}\right]$, HAADF and supplementary HRTEM on Co@SnPtAu, TEM on efforts for $\mathrm{Au}$ and Pt shell thickening, UV-Vis spectra of Co@SnAu after efforts for Au shell thickening, TEM of the effect of water on Co@SnAu and Co@SnPt nanorods, STEM-EDX showing the complementary presence of Au and Pt in Co@SnPtAu nanorods, TEM, EDX of Co@SnPtAu nanorods in water, TEM, EDX results on Co@SnAuPt nanorods, XRD results on 
powders of core-shell nanorods, complementary XANES and EXAFS data, TEM, EDX EXAFS and XPS data on the Co@Sn intermediate, TEM of all core-shell samples before and after shell growth. This material is available free of charge via the Internet at http://pubs.acs.org.

\author{
AUTHOR INFORMATION \\ Corresponding Author \\ *Katerina Soulantica \\ *e-mail: ksoulant@insa-toulouse.fr
}

\title{
Author Contributions
}

The manuscript was written through contributions of all authors. All authors have given approval to the final version of the manuscript.

\section{ACKNOWLEDGMENT}

The authors thank the European Commission for the FP7 NAMDIATREAM project (EU NMP4-LA-2010-246479), the European Commission and the Région Midi-Pyrénées for the POCTEFA Interreg project (MET-NANO EFA 17/08) and the French Research National Agency (ANR) for the project CARMA (ANR-09-BLAN-0804). This study has been partially supported through the grant NEXT n ANR-10-LABX-0037 in the framework of the "Programme des Investissements d'Avenir" and has received funding from the European Union Seventh Framework Programme under Grant Agreement 312483 - ESTEEM2 (Integrated Infrastructure Initiative-I3). TEMSCAN service is acknowledged for the routine TEM and EDX. G.V.T. and S.B. acknowledge financial support from European Research Council (ERC Advanced Grant \# 
24691-COUNTATOMS, and ERC Starting Grant \# 335078-COLOURATOMS). X-ray

absorption spectroscopy studies by JTM and JRG were supported by the Institute for Atomefficient Chemical Transformations (IACT), an Energy Frontier Research Center funded by the US Department of Energy, Office of Science, Office of Basic Energy Sciences. Use of the Advanced Photon Source is supported by the U.S. Department of Energy, Office of Science, and Office of Basic Energy Sciences, under Contract DE-AC02-06CH11357. MRCAT operations are supported by the Department of Energy and the MRCAT member institutions.

\author{
ABBREVIATIONS \\ Co-NRs, Co single-crystalline nanorods; $h c p$, hexagonal close packed; fcc, face centered cubic; \\ POC, point-of-care; HDA, hexadecylamine; Me, methyl; COD, cyclooctadiene; $\mathrm{PPh}_{3}$, \\ triphenylphosphine; TEM, Transmission Electron Microscopy; HRTEM, High Resolution \\ Transmission Electron Microscopy; STEM-HAADF, Scanning Transmission Electron -High- \\ Angle Annular Dark-Field; STEM-EDX, Scanning Transmission Electron Microscopy-Energy \\ Dispersive X-ray; XRD, X-Ray Diffraction; FFT, the Fast Fourier Transform; XAS, X-Ray \\ Absorption Spectroscopy; XANES, X-ray Absorption Near Edge Structure; EXAFS, Extended \\ X-Ray Absorption Fine Structure; XPS, X-ray Photoelectron Spectroscopy; EB, exchange bias; \\ FC, field cooling; ZFC, zero field cooling; VSM, Vibrating Sample Magnetometry; LSPR, \\ Localized Surface Plasmon Resonance.
}

\title{
REFERENCES
}

1. Sun, S.; Murray, C. B.; Weller, D.; Folks, L.; Moser, A. Monodisperse FePt Nanoparticles and Ferromagnetic FePt Nanocrystal Superlattices. Science 2000, 287, 1989-1992. 
2. Frey, N. A.; Peng, S.; Cheng, K.; Sun, S. Magnetic Nanoparticles: Synthesis, Functionalization and Applications in Bioimaging and Magnetic Energy Storage. Chem. Soc. Rev. 2009, 38, 2532-2542.

3. Lu, A.-H.; Salabas, E. L.; Schlüth, F. Magnetic nanoparticles: Synthesis, protection, Functionalization and Application. Angew. Chem. Int. Ed. 2007, 46, 1222-1244.

4. Ho, D.; Sun, X.; Sun, S. Monodisperse Magnetic Nanoparticles for Theranostic Applications. Acc. Chem. Res. 2011, 44, 875-882.

5. Hyeon, T. Chemical Synthesis of Magnetic Nanoparticles. Chem. Commun. 2003, 927-934.

6. Diehl, G. A.; Doyle, J.-Y.; Heath, J. R.; Held, G. A.; Doyle, H.; Sun, S.; Murray, C. B. Crystalline, Shape, and Surface Anisotropy in Two Crystal Morphologies of Superparamagnetic Cobalt Nanoparticles by Ferromagnetic Resonance. J. Phys. Chem. B 2001, 105, 7913-7919.

7. Thurn-Arbrecht, T.; Schotter, J.; Kästle, G. A.; Emley, N.; Shibauchi, T.; Krusin-Elbaum. L.; Guarini, K.; Black, C. T.; Tuominen, M. T.; Russel, T. P. Ultrahigh-Density Nanowire Arrays Grown in Self-Assembled Diblock Copolymer Templates. Science 2000, 290, 2126-2129.

8. Maurer, T.; Ott, F.; Chaboussant, G.; Soumare, Y.; Piquemal, J.-Y.; Viau, G. Magnetic Nanowires as Permanent Magnet Materials. Appl. Phys. Lett. 2007, 91, 172501.

9. Dumestre, F.; Chaudret, B.; Amiens, C.; Fromen, M.-C.;Casanove, M.-J.; Renaud, P.; Zurcher, P. Shape Control of Thermodynamically Stable Cobalt Nanorods Through Organometallic Chemistry Angew. Chem. Int. Ed. 2002, 41, 4286-4289.

10. Wetz, F.; Soulantica, K.; Respaud, M.; Falqui, A.; Chaudret, B. Synthesis and Magnetic Properties of Co Nanorod Superlattices. Mater. Sci. Eng. C 2007, 27, 1162-1166. 
11. Soulantica, K.; Wetz, F.; Maynadié, J.; Falqui, A.; Tan, R. P.; Blon, T.; Chaudret, B.; Respaud, M. Appl. Phys. Lett. 2009, 95, 152504.

12. Gutfleisch, O.; Willard, M.A.; Brück, E.; Chen, C.H.; Sankar, S.G.; Liu, J.P. Magnetic Materials and Devices for the 21st Century: Stronger, Lighter, and More Energy Efficient. Adv. Mater. 2011, 23, 821-842.

13. Peng, S.; Wang, C.; Xie, J.; Sun, S. Synthesis and Stabilization of Monodisperse Fe Nanoparticles. J. Am. Chem. Soc. 2006, 128, 10676-10677.

14. Yoon, T.-J., Lee, H., Shao, H.; Weissleder, R. Highly Magnetic Core-Shell Nanoparticles with a Unique Magnetization Mechanism. Angew. Chem. Int. Ed. 2011, 50, 4663-4666.

15. Seo, W. S. Lee, J. H.; Sun, X.; Suzuki, Y.; Mann, D.; Liu, Z.; Terashima, M.; Yang, P. C.; McConnel, M. V. et al. FeCo/Graphitic-Shell Nanocrystals as Advanced Magnetic-ResonanceImaging and Near-Infrared Agents. Nature Mater. 2006, 5, 971-976.

16. Grass, R. N.; Athanassiou, E. K.; Stark, W.J. Covalently Functionalized Cobalt Nanoparticles as a Platform for Magnetic Separations in Organic Synthesis. Angew. Chem. Int. Ed. 2007, 46, 4909-4912.

17. Levin, C. S.; Hofmann, C.; Ali, T. A.; Kelly, A. T.; Morosan, E.; Nordlander, P.; Whitmire, K. H.; Halas, N. J. Magnetic-Plasmonic Core-Shell Nanoparticles. ACS Nano 2009, 3, 13791388.

18. Fan, Z.; Shelton, M.; Singh, A.K.; Senapati, D.; Khan, S. A.; Ray, P. C. Multifuctional Plasmonic Shell-Magnetic Core Nanoparticles for Targeted Diagnostics, Isolation, and Photothermal Destruction of Tumor Cells. ACS Nano 2012, 6, 1065-1073. 
19. Elghanian, R.; Storhoff, J. J.; Mucic, R. C.; Letsinger, R. L; Mirkin, C. A. Selective Colorimetric Detection of Polynucleotides Based on the Distance-Dependent Optical Properties of Gold Nanoparticles. Science 1997, 277, 1078-1081.

20. Guo, S.; Zhang, S.; Sun, S. Tuning Nanoparticle Catalysis for the Oxygen Reduction Reaction. Angew. Chem. Int. Ed. 2013, 52, 8526-8544.

21. Sasaki, K.; Naohara, H.; Cai, Y.; Choi, Y.M.; Lui, P.; Vukmirovic, M. B.; Wang, J. X.; Adzic, R. R. Core-Protected Platinum Monolayer Shell High-Stability Electrocatalysts for FuelCell Cathodes. Angew. Chem. Int. Ed. 2010, 49, 8602-8607.

22. Liu, Y.; Li, D.; Sun, S. Pt-Based Composite Nanoparticles for Magnetic, Catalytic, and Biomedical Applications. J. Mater. Chem. 2011, 21, 12579-12587.

23. Sapsford, K. E.; Algar, W. R.; Berti, L.; Boeneman Gemmill, K.; Casey, B. J.; Oh, E.; Stewart, M. H.; Medintz, I. L. Functionalizing Nanoparticles with Biological Molecules: Developing Chemistries that Facilitate Nanotechnology. Chem. Rev. 2013, 113, 1904-2074.

24. Chaudhuri, R. G.; Paria, S. Core/Shell Nanoparticles: Classes, Properties, Synthesis Mechanisms, Cheracterization, and Applications Chem. Rev. 2012, 112, 2373-2433.

25. Habas, S. E.; Lee, H.; Radmilovic, V.; Somorjai, G. A.; Yang, P. Shaping Binary Metal Nanocrystals Through Epitaxial Seeded Growth. Nature Mater. 2007, 6, 692-697.

26. Tao, A. R.; Habas, S.; Yang, P. Shape Control of Colloidal Metal Nanocrystals. Small 2008, 3, 310-325.

27. Wang, L.-L.; Johnson, D. D. Predicted Trends of Core-Shell Preferences for 123 Late Transition-Metal Binary-Alloy Nanoparticles. J. Am. Chem. Soc. 2009, 131, 14023-14029. 
28. Min, Y.; Kwak, J.; Soon, A.; Jeong, U. Nonstoichiometric Nucleation and Growth of Multicomponent Nanocrystals in Solution. Acc. Chem. Res. 2014, 47, 2887-2893.

29. Carbone, L.; Cozzoli, P. D. Colloidal Heterostructured Nanocrystals: Synthesis and Growth Mechanisms. NanoToday 2010, 5, 449-493.

30. Feng, Y.; He, J.; Tay, Y. Y.; Sun, H.; Zhu, L.; Chen, H. An Unconventional Role of Ligand in Continuously Tuning of Metal-Metal Interfacial Strain. J. Am. Chem. Soc. 2012, 134, 20042007.

31. Lee, W.; Kim, M. G.; Choi, J.; Park, J.-I.; Ko, S. J.; Oh, S .J.; Cheon, J. RedoxTransmetalation Process as a Generalized Synthetic Startegy for Core-Shell Magnetic Nanoparticles. J. Am. Chem. Soc. 2005, 127, 16090-16097.

32. Lu, Z.; Prouty, M. D.; Guo, Z.; Golub, V. O.; Kumar, C. S. S. R.; Lvov, Y. M. Magnetic Switch of Permeability for Polyelectrolyte Microcapsules Embedded with Co@Au Nanoparticles. Langmuir 2005, 21, 2042-2050.

33. Bao, Y.; Calderon, H.; Krishnan, K. M. Synthesis and Characterization of MagneticOptical Co-Au Core-Shell Nanoparticles. J. Phys. Chem. C 2007, 111, 1941-1944.

34. Yager, P.; Domingo, G. J.; Gerdes, J. Point-of-Care Diagnostics for Global Health. Annu. Rev. Biomed. Eng. 2008, 10, 107-144.

35. Schrittwieser, S. Ludwig, F.; Dieckhoff, J.; Soulantica, K.; Viau, G.; Lacroix, L.-M. Lentijo Mozo, S.; Boubekri, R.; Maynadié, J. et al. Modeling and Development of a Biosensor Based on Optical Relaxation Measurements of Hybrid Nanoparticles. ACS Nano 2012, 6, 791801. 
36. Liakakos, N.; Blon, T.; Achkar, C.; Vilar, V.; Cormary, B.; Tan, R. P.; Benamara, O.; Chaboussant, G.; Ott, F.; Warot-Fonrose, B.; et al. Solution Epitaxial Growth of Cobalt Nanowires on Crystalline Substrates for Data Storage Densities Beyond 1Tbit/in². Nano Lett. 2014, 14, 3481-3486.

37. Mertelj, A.; Lisjak, D.; Drofenik, M.; Copic, M. Ferromagnetism in Suspensions of Magnetic Platelets in Liquid Crystal. Nature 2013, 504, 237-241.

38. Salgueirino-Maceira, V.; Liz-Marzán, L.; Farle, M. Water-Based Ferrofluids from FexPt1-x Nanoparticles Synthesized in Organic Media. Langmuir 2004, 20, 6946-6950.

39. Soulantika, A.; Lentijo-Mozo, S.; Hungria-Hernandez, M.-T.; Tan, R. P.; Gatel, C. Magnetic Nano-Objects Covered with a Metallic Envelope. Patent № WO/2014 102496.

40. Shylesh, S.; Schünemann, V.; Thiel, W. R. Magnetically Separable Nanocatalysts: Bridges Between Homogeneous and Heterogeneous Catalysis. Angew. Chem. Int. Ed. 2010, 49, 34283459.

41. Rossi, L. M.; Costa, N. J. S.; Silva, F. P.; Wojcieszak, R. Magnetic Nanomaterials in Catalysis: Advanced Catalysts for Magnetic Separation and Beyond. Green Chem. 2014, 16, 2906-2933.

42. Wetz, F. Soulantica, K.; Falqui, A.; Respaud, M.; Snoeck, E.; Chaudret, B. Hybrid Co-Au nanorods: Controlling Au Nucleation and Location. Angew. Chem. Int. Ed. 2007, 46, 7079-7081.

43. Markov, I.V. Crystal Growth for Beginners, $2^{\text {nd }}$ Edition., World Scientific Publishing Co, Singapore, 2003, Chapter 4. ) 
44. Peng, Z.; Yang, H. Designer Platinum Nanoparticles: Control of Shape, Composition in Alloy, Nanostructure and Electrocatalytic Property. Nano Today 2009, 4, 143-164.

45. Kanatzidis, M. G.; Pöttgen, R.; Jeitschko, W. The Metal Flux: A Preparative Tool for the Exploration of Intermetallic Compounds. Angew. Chem. Int. Ed. 2005, 44, 6996-7023.

46. Cable, R. E.; Schaak, R. E. Low-Temperature Solution Synthesis of Nanocrystalline Binary Intermetallic Compounds Using the Polyol Process. Chem. Mater. 2005, 17, 6835-6841.

47. Chou, N. H.; Schaak, R. E. A Library of Single-Crystal Metal-Tin Nanorods: Using Diffusion as a Tool for Controlling the Morphology of Intermetallic Nanocrystals. Chem. Mater. 2008, 20, 2081-2085.

48. Somodi, F.; Peng, Z.; Getsoian, A. B.; Bell, A. T. Effects of the Synthesis Parameters on the Size and Composition of Pt-Sn Nanoparticles Prepared by the Polyalcohol Reduction Method. J. Phys. Chem. C 2011, 115, 19084-19090.

49. McBride, J.; Treadway, J.; Feldman, L. C.; Pennycook, S. J.; Rosenthal, S. J. Structural Basis for Near Unity Quantum Yield Core/Shell Nanostructures. Nano Lett. 2006, 6, 1496-1501.

50. Zhang, S.; Hao, Y.; Su, D.; Doan-Nguyen, V. V. T.; Wu, Y.; Li, J.; Sun, S., Murray, C. B. Monodisperse Core/Shell Ni/FePt Nanoparticles and Their Conversion to Ni/Pt to catalyze Oxygen Reduction. J. Am. Chem. Soc. 2014, 136, 15921-15924.

51. Co [uvtw] (hkil) // M [u'v'w'] (h'k'l') means that the Co (hkil) plane is parallel to the Au (h'k'l') plane and that for these planes the Co [uvtw] direction is parallel to the M [u'v'w'] direction. 
52. Bond, G.C. The Electronic Structure of Platinum-Gold Alloy Particles. Platimun Metals Rev. 2007, 51, 63-68.

53. Olmstead, M. M.; Power, P. P. Structural Studies of Tin(II) and Lead(II) Dimethylamides: X-Ray Crystal Structure of $\left[\mathrm{Sn}\left(\mathrm{NMe}_{2}\right)_{2}\right]_{2}$ and Isolation of its Lead Analogue. Inorg. Chem. 1984, 23, 413-415.

54. Dahéron, L.; Dedryvère, R.; Martinez, H.; Ménétier, M.; Denage, C.; Delmas, C.; Gonbeau, D. Transfer Mechanisms upon Lithium Deintercalation from $\mathrm{LiCoO}_{2}$ to $\mathrm{CoO}_{2}$ Investigated by XPS. Chem. Mater. 2008, 20, 583-590.

55. Verelst, M; Ould Ely, T.; Amiens, C.; Snoeck, E.; Lecante, P.; Mosset, A.; Respaud, M.; Broto, J.-M.; Chaurdet, B. Synthesis and Characterization of $\mathrm{CoO}, \mathrm{Co}_{3} \mathrm{O}_{4}$, and Mixed $\mathrm{Co} / \mathrm{CoO}$ Nanoparticles. Chem. Mater. 1999, 11, 2702-2708.

56. Sun, H.; Chen, B.; Jiao, X.; Jiang, Z.; Qin, Z.; Chen, D. Solvothermal Synthesis of Tunable Electroactive Magnetite Nanorods by Controlling the Side Reaction. J. Phys. Chem. C 2012, $116,5476-5481$.

57. Palchoudhury, S.; An, W.; Xu, Y.; Qin, Y.; Zhang, Z.; Chopra, N.; Holler, R. A.; Turner, C. H.; Bao, Y. Synthesis and Growth Mechanism of Iron Oxide Nano Whiskers. Nano Lett. 2011, 11, 1141-1146.

58. Pondman, K. M.; Maijenburg, A. W.; Celikkol, F. B.; Pathan, A. A.; Kishore, U., ten Haken, B.; ten Elshof, J. E. Au coated Ni Nanowires with Tuneable Dimensions for Biomedical Applications. J. Mater. Chem. B 2013, 1, 6129-6136. 
59. Suda, M.; Einaga, Y. Sequential Assembly of Phototunable Ferromagnetic Ultrathin Films with Perpendicular Magnetic Anisotropy. Angew. Chem. Int. Ed. 2009, 48, 1754-1757.

60. Schlossmacher, P.; Klenov, D. O.; Freitag, B.; Von Harrach, H. S. Enhanced Detection Sensitivity with a New Windowless XEDS System for AEM Based on Silicon Drift Detector Technology. Microscopy Today 2010, 18, 14-20.

61. Ressler T. WinXAS: A Program for X-ray Absorption Spectroscopy Data Analysis Under MS-Windows. J Synchrotron Radiat. 1998, 5, 118-122.

62. Rehr, J. J.; Deleon, J. M.; Zabinsky S. I.; Albers R. C. Theoretical X-Ray Absorption Fine Structure Standards. J. Am. Chem. Soc., 1991, 113, 5135-5140.

63. Shirley, D. A. High-Resolution X-Ray Photoemission Spectrum of the Valence Bands of Gold. Phys. Rev. B 1972, 5, 4709-4714.

64. Scofield, J. H. Hartree-Slater Subshell Photoionization Cross-Sections at 1254 and 1487 eV. J. Electron Spectrosc. Relat. Phenom. 1976, 8, 129-137. 